\title{
A little frustration to sharpen the metamaterial
}

\author{
Non-orientable order and non-Abelian response in frustrated \\ metamaterials \\ Authors: Xiaofei Guo, Marcelo Guzman, David Carpentier, Denis Bartolo, and \\ Corentin Coulais \\ arXiv:2111.13933
}

\section{Recommended with a Commentary by Anton Souslov, University of Bath}

\author{
People need trouble - a little \\ frustration to sharpen the spirit on, \\ toughen it.
}

William Faulkner

We often become frustrated when faced with many equivalent choices, for example when picking flavors in an ice cream shop. Similarly, materials become frustrated when their constituents can locally arrange themselves in one of several equivalent ways. At the macroscale, the matter that emerges from these local arrangements has many ground states with degenerate energies. Frustrated materials are as common as window glass - or more precisely, atomic and molecular glasses such as silica as well as so-called spin glasses in magnetic systems. In all of these cases, the frustration is local and geometric: for example, silica molecules can bind to each other at several energetically equivalent angles. Moreover, frustration tests the foundations of physics. Ground-state degeneracy violates the third law of thermodynamics, because entropy will not vanish even at zero temperature.

In their recent preprint, Guo et al. [1] design materials in which frustration becomes naturally wedded to global, topological properties. Although locally, it may seem that every element can satisfy its preferred configuration, globally, it is impossible for every element to simultaneously satisfy this configuration. This incompatibility is imposed by a topological constraint: an unchangeable property of the material as a whole, rather than any one angle. One example is a configuration of interlocking gears on a ring: since two neighboring gears have to counter-rotate, a ring composed of an even number of gears can move, but a ring with an odd number of gears is locked into place by frustration (see Fig. 1 and for a more detailed explanation, the YouTube video in Ref. [2]). This physics plays out in a variety of contexts, from active fluid flow $[3,4,5]$ to molecular rings [6] and bead packings [7].

The topological feature that Guo et al. [1] exploit is orientability. Heuristically, an orientable surface is one that encloses an "inside" volume and separates it from an "outside." 
(a)

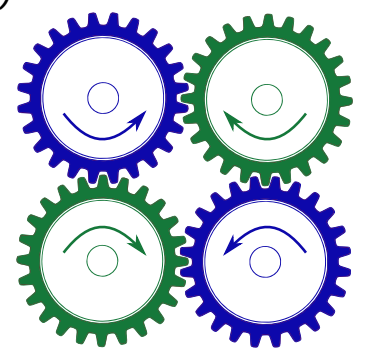

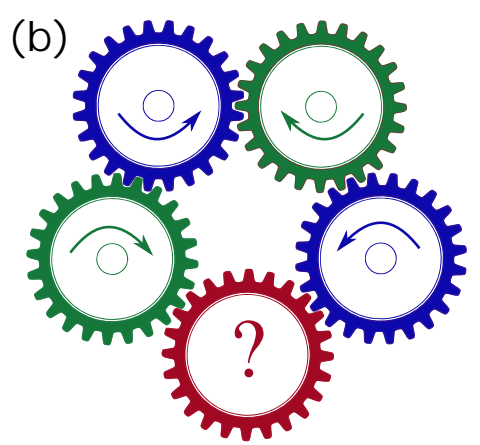

Figure 1: Rings composed of (a) an even and (b) an odd number of gears. When the number of gears is even, a checkerboard patterns allows all of the gears to rotate. For an odd number of gears, frustration locks the assembly in place.

For example, a beach ball can be inflated precisely because a sphere is orientable: the inside of a beach ball is enclosed and the air cannot escape. Some surfaces such as a Möbius strip (Fig. 2) are non-orientable, which translates into surprising mechanics. When Guo et al. [1] squeeze a Möbius strip from all sides, they show that a part of the shape remains undeformed because it does not know which way to buckle.

Although the ideas of topology and non-orientability appear abstract, Guo et al. [1] bring them into the material world using state-of-the-art 3D printing of mechanical meta-surfaces and meta-rings. In the ring, Fig. 3, there are two rows with equal numbers of squares, so that the squares in the outer row are bigger than the squares in the inner row. When the material is squeezed, each square rotates to shorten the distance to its neighbors. Each square can decide to rotate clockwise or counter-clockwise, and two neighbors want to rotate in opposite directions - much like the example of the gears. On a line, this would form a perfectly ordered checkerboard pattern of alternating directions of rotation. But on a ring with an odd number of squares, this is impossible: two of the squares will not know which way to rotate and will be frustrated. Just like for the orientation of a Möbius strip, the sense
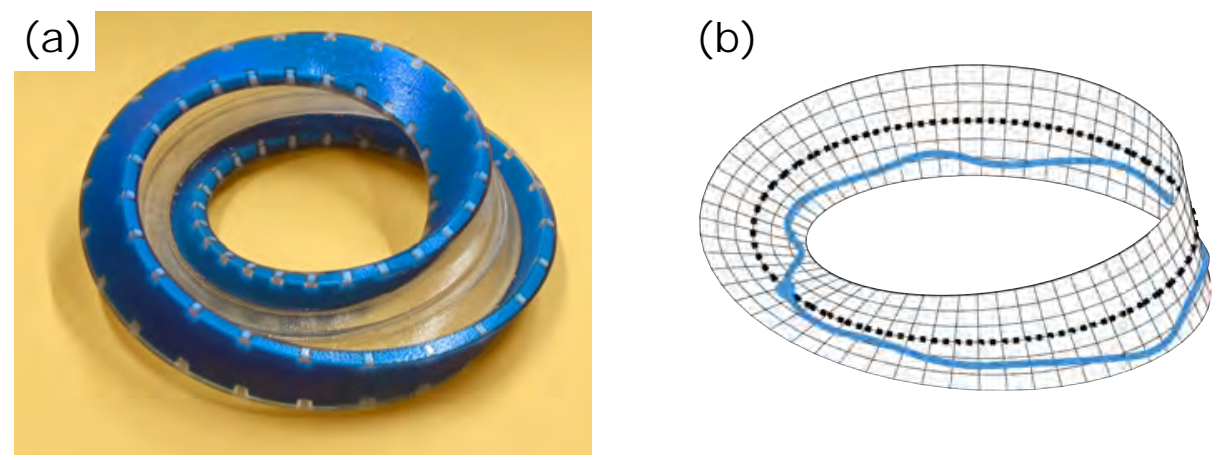

Figure 2: Soft Möbius strip. (a) A Möbius strip 3D printed using a flexible material. (b) An illustration of a Möbius strip showing non-orientability. If an ant traverses once the drawn path, the ant will end up on the opposite side of the strip. Image courtesy of Xiaofei Guo, see also Ref. [1]. 

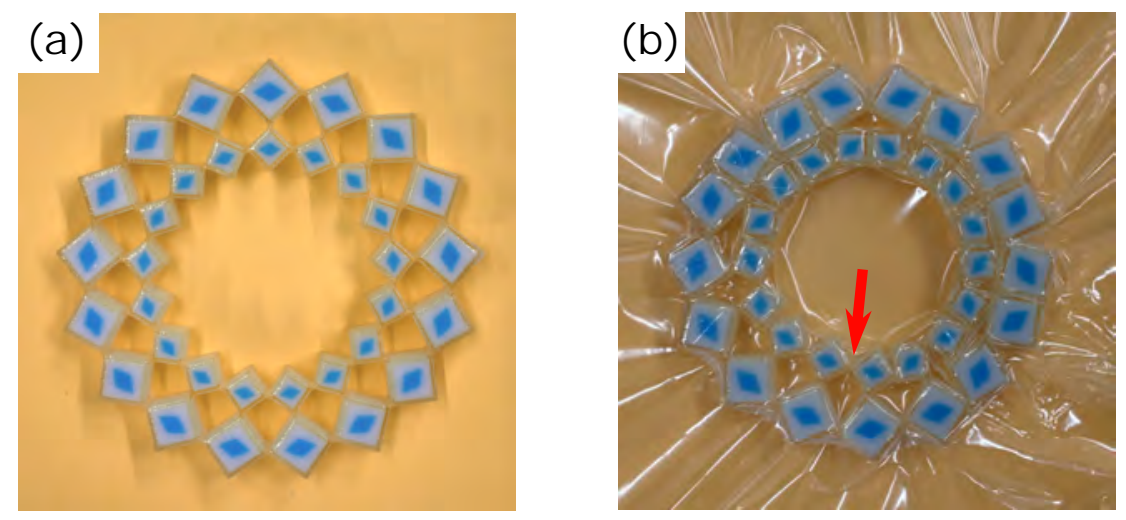

Figure 3: Non-orientable mechanical metamaterial. (a) 3D printed sample of a non-orientable metamaterial ring. There is an odd number of squares in each row. (b) Under compression in a vacuum bag, every square rotates counter to its neighbors. The ring is frustrated because there are an odd number of squares, which leads to a defect (shown here on the bottom side with an arrow). Image courtesy of Xiaofei Guo, see also Ref. [1].

of rotation for each square cannot be globally undefined. To figure out what happens, Guo et al. [1] used simple and effective experiments: they squeezed the meta-ring uniformly inside a vacuum bag! The frustrated squares, shown with an arrow on the bottom in Fig. 3(b), did not rotate at all.

Introducing a new materials design principle of topological frustration is exciting, because we never know where these discoveries may lead. Guo et al. [1] present one possible application: mechanical computers. The defects resulting from frustration can be thought of as bits in a computer, and manipulating several of these bits at once allows for simple arithmetic operations to be performed. As a demonstration, their manuscript shows a simple flip-flop circuit that can be used for sequential logic.

Topological design has become a staple of modern science from corralling electrons, phonons, and photons in edge states $[8,9,10,11]$ to topology optimization in engineered structures [12]. Exploring topology in non-orientable mechanics could unlock many more suprises for how to design devices based on metamaterials, focusing more on the global configuration of the constituent elements than the structure of a single meta-atom. There are many equally exciting directions to pursue, but once we persevere through this frustrating choice, we are bound to be well rewarded-just like back in the ice cream shop!

\section{References}

[1] Xiaofei Guo, Marcelo Guzman, David Carpentier, Denis Bartolo, Corentin Coulais. Nonorientable order and non-Abelian response in frustrated metamaterials, arXiv:2111.13933.

[2] Are odd-numbered mobius-loop cogs possible? YouTube (2019)

https ://www . youtube. com/watch?v=nWQwrU1qUrc 
[3] Hugo Wioland, Francis G. Woodhouse, Jörn Dunkel, and Raymond E. Goldstein Ferromagnetic and antiferromagnetic order in bacterial vortex lattices. Nature Physics 12, 341 (2016).

[4] Anton Souslov, Benjamin C. van Zuiden, Denis Bartolo, and Vincenzo Vitelli. Topological sound in active-liquid metamaterials. Nature Physics 13, 1091 (2017).

[5] Amélie Chardac, Ludwig A. Hoffmann, Yoann Poupart, Luca Giomi, and Denis Bartolo. Topology-Driven Ordering of Flocking Matter. Physical Review X 11, 031069 (2021)

[6] Michael L. Baker et al. A classification of spin frustration in molecular magnets from a physical study of large odd-numberedmetal, odd electron rings. Proceedings of the National Academy of Sciences 109, 19113 (2012).

[7] Yilong Han, Yair Shokef, Ahmed M. Alsayed, Peter Yunker, Tom C. Lubensky, and Arjun G. Yodh Geometric frustration in buckled colloidal monolayers. Nature 456, 898 (2008).

[8] M. Z. Hasan \& C. L. Kane Colloquium: topological insulators. Reviews of Modern Physics 82, 3045 (2010).

[9] Suraj Shankar, Anton Souslov, Mark J. Bowick, M. Cristina Marchetti, Vincenzo Vitelli. Topological active matter, arXiv:2010.00364.

[10] H. Nassar et al. Nonreciprocity in acoustic and elastic materials. Nature Reviews Materials 5, 667-685 (2020).

[11] T. Ozawa et al. Topological photonics. Reviews of Modern Physics 91, 15006 (2019).

[12] Hans Eschenauer and Niels Olhoff. Topology optimization of continuum structures: a review. Applied Mechanics Reviews 54, 331-390 (2001). 